\title{
Editorial Introduction to the Special Issue: Interpretation of Solid-State Terahertz Spectra
}

\author{
Michael T. Ruggiero ${ }^{1}$ \\ Published online: 26 September 2020 \\ (C) Springer Science+Business Media, LLC, part of Springer Nature 2020
}

Terahertz spectroscopy has proven to be an especially powerful technique for the analysis of condensed-phase dynamics. The origin of this utility lies with its direct probe of weak, often intermolecular, forces - forces that are responsible for dictating a number of important material phenomena ranging from thermal expansion to polymorphic stability. However, the interpretation of the terahertz spectra of solid-state materials represents a particularly challenging task, as the weak interactions - and associated terahertz dynamics - are highly dependent on the bulk atomic and molecular structures, with no functional group-specific transitions, unlike mid-infrared techniques. Fortunately, over the last decade, advances in quantum mechanical software packages and computational technologies have opened the door for simulations that achieve unprecedented numerical accuracy, enabling the faithful reproduction of lowfrequency dynamics and associated phenomena. When performed appropriately (i.e., with periodic boundary condition simulations), the combination of low-frequency vibrational spectroscopy and spectral assignment using ab initio methods enables incredible insight into the fundamental forces that shape the properties of condensed-phase materials. This special issue contains contributions highlighting the current state-of-the-art in this respect, featuring work that provides a strong foundation and introduction into this important area of terahertz science, ultimately serving as a valuable resource for both experts and non-experts. It contains 9 invited articles, the content of which is briefly outlined below.

1. Crystalline Molecular Standards for Low-Frequency Vibrational Spectroscopies by S. J. Dampf and T. M. Korter (Syracuse University, USA) explores a set of molecular crystals using experimental terahertz time-domain and low-frequency Raman spectroscopies, as well as the corresponding assignment using periodic density functional theory simulations, to generate a suggested set of standards for future reference.

2. Vibrational Response of Felodipine in the THz Domain: Optical and Neutron Spectroscopy versus Plane-Wave DFT Modeling by K. Łuczyńska, K. Drużbicki, T. Runka, N. Pałka, and J. Węsicki (a collaboration between Adam Mickiewicz University, Poland;

Michael T. Ruggiero

michael.ruggiero@uvm.edu

1 Department of Chemistry, University of Vermont, Burlington, VT 05405, USA 
Frank Laboratory of Neutron Physics, Russian Federation; Poznan University, Poland; and the Military University of Technology, Poland) applies computational periodic planewave density functional theory and ab initio molecular dynamics simulations to study a polymorph of the active pharmaceutical ingredient, felodipine, in conjunction with terahertz, low-frequency Raman, and inelastic neutron scattering spectroscopies.

3. Time-domain Terahertz Spectroscopy and Solid-State Density Functional Theory Analysis of p-Nitrophenol Polymorphs by T. H. da Silva, N. R. Rexrode, and M. D. King (Boise State University, USA) studies the polymorphic phase transition in a molecular crystal using terahertz spectroscopy and density functional theory simulations, highlighting the effect of thermal expansion on the terahertz spectral features.

4. Assignment of Terahertz, Modes in Hydroquinone Clathrates by W. Zhang, Z. Song, M. T. Ruggiero, and D. M. Mittleman (a collaboration between Brown University and the University of Vermont, USA) combines pressure- and temperature-dependent terahertz time-domain spectroscopy experiments and anharmonic-corrected periodic density functional theory simulations to assign the spectra of porous molecular crystals, hydroquinone clathrates, with various guest molecules.

5. Terahertz Spectroscopy and Density Functional Theory Investigation of the Dipeptide LCarnosine by J. Neu and C. A. Schmuttenmaer (Yale University, USA) performs an experimental and solid-state density functional theory characterization of a biologically relevant condensed-phase dipeptide, L-carnosine.

6. High-Resolution THz Spectroscopy and Solid-State Density Functional Theory Calculations of Polycyclic Aromatic Hydrocarbons by F. Zhang, H.-W. Wang, K. Tominaga, M. Hayashi, and T. Sasaki (a collaboration between Kobe University, Japan; National Taiwan University, Taiwan; and Shizuoka University, Japan) utilizes high-resolution broadband terahertz spectroscopy to measure the terahertz spectra of nine polycyclic aromatic hydrocarbons, along with a corresponding assignment using solid-state density functional theory simulations.

7. Tracking the Acceleration of Hydration of $\beta-C 2 S$ due to Nanosilica Incorporation using THz Spectroscopy by S. Ray, N. Devi, J. Dash, S. Sasmal, and B. Pesala (Council of Scientific and Industrial Research, India) utilizes terahertz spectroscopy for tracking hydration in silica nanoparticles and highlights a possible mechanism based on the spectroscopy and microscopy experiments.

8. Assessing the Performance of Density Functional Theory Methods on the Prediction of Low-Frequency Vibrational Spectra by P. A. Banks, Z. Song, and M. T. Ruggiero (University of Vermont, USA) highlights the performance of periodic density functional theory, specifically the role of basis set and functional, on the prediction of the terahertz spectra of three molecular crystals.

9. Exploring the Reliability of DFT Calculations of the Infrared and Terahertz Spectra of Sodium Peroxodisulfate by J. Kendrick and A. D. Burnett (University of Leeds, UK) thoroughly investigates the ability of various density functional theory methods to 
reproduce the terahertz spectra of solid-state sodium peroxodisulfate. Note: This article was erroneously published in a previous issue (J. Infrared Millim. Terahertz Waves, 41(4), 382-413, 2020).

Publisher's Note Springer Nature remains neutral with regard to jurisdictional claims in published maps and institutional affiliations. 